\title{
NIVELES DE INTERSUBJETIVIDAD Y CONCEPTUALIZACIÓN GRAMATICAL: EL CASO DEL CAMBIO SEMÁNTICO DE LA PREPOSICIÓN ANTE*
}

\author{
LEVELS OF INTERSUBJECTIVITY AND GRAMMATICAL \\ CONCEPTUALIZATION: THE SEMANTIC CHANGE OF THE \\ PREPOSITION ANTE
}

\author{
ENRIQUE HUELVA UNTERNBÄUMEN \\ Universidad de Brasilia (UnB). Brasilia, Brasil \\ huelva@unb.br
}

\section{RESUMEN}

Esta investigación trata de la intersubjetividad como categoría codificada gramaticalmente. Complementando estudios anteriores, pretendemos demostrar que la gramática no codifica dicha categoría de forma holística, sino que, por el contrario, nos permite distinguir entre diferentes tipos y grados de complejidad de la intersubjetividad. Esta diferenciación se manifiesta tanto sincrónicamente, en la distribución del uso y el potencial semántico de elementos lingüísticos que actúan en el mismo dominio conceptual, como diacrónicamente, en la evolución semántica de un mismo elemento. Nuestro objeto de análisis concreto es la preposición ante de la lengua española.

Palabras clave: Codificación gramatical, evolución semántica, intersubjetividad, potencial semántico.

\begin{abstract}
This investigation deals with intersubjectivity as a grammatically codified category. Complementing previous studies, we intend to demonstrate that grammar does not codify that category in a holistic manner, but rather, to the contrary, permits us to distinguish among different type and degrees of complexity of intersubjectivity. This differentiation manifests itself both synchronically, in the distribution of the use and semantic potential of linguistic elements acting in the same conceptual domain, and diachronically, in the
\end{abstract}

\footnotetext{
" Proyecto "Intersubjetividad y gramática" vinculado al Programa de Posgrado del Instituto de Letras de la Universidad de Brasilia.
} 
RLA. Revista de Lingüística Teórica y Aplicada, 52 (2), II Sem. 2014

semantic evolution of a single element. Our concrete object of analysis is the Spanish proposition ante.

Keywords: Grammatical codification, semantic evolution, intersubjectivity, semantic potencial.

Recibido: 21.03.2014. Aprobado: 01.08.2014.

\section{INTRODUCCIÓN}

T a intersubjetividad es una de las características esenciales de la condición hu_mana. Como señala Gallagher (2012: 2-3), nacemos en un mundo de la vida que desde el principio ya está poblado por otros y construido por sus acciones. Antes de que tengamos la posibilidad y las condiciones necesarias para ubicarnos en el mundo, antes de que podamos posicionarnos en el mundo como sujetos, de poder ser, estar y actuar en el mundo, nuestras capacidades para realizar todo esto han sido ya moldeadas por otros seres humanos que se encuentran con nosotros antes incluso de nuestro nacimiento y que nos hablan antes de que tengamos la posibilidad de responder, que interactúan con nosotros antes de que tengamos las condiciones de actuar por cuenta propia. Subjetividad significa, desde el primer momento, intersubjetividad.

La intersubjetividad, en el sentido fenomenológico que acabamos de sugerir y que subyacerá a las reflexiones que desarrollaremos en las próximas páginas, mantiene una doble relación con la lengua. Por una parte constituye uno de sus principales requisitos, tanto filo- como ontogénicamente. Una lengua natural es un sistema simbólico convencional y como tal su dominio requiere la existencia de un conocimiento compartido entre todos los miembros de la comunidad que usan una determinada lengua (Itkonen, 2008; Zlatev, 2007; 2008: 232-237). Este conocimiento compartido suele ser caracterizado como un conocimiento de tercer orden yo sé que tú sabes que yo sé $X$ y es considerado como uno de los niveles más complejos de intersubjetividad (Zlatev, 2008: 234-237). Con la adquisición del lenguaje, por otra parte, desarrollamos una capacidad meta-representacional, en el sentido de que su uso nos permite conceptuarnos a nosotros mismos y a los otros como seres intersubjetivos (Zlatev, 2008: 235-236). El léxico y, en particular, la gramática, codifican diferentes tipos y niveles de intersubjetividad, diferentes configuraciones de intersubjetividad (Huelva Unternbäumen 2013a). Esto es, la intersubjetividad forma parte -en mayor o menor medida (Huelva Unternbäumen, 2013a) - de la estructura semántica de muchos elementos lingüísticos.

El segundo tipo de relación es el que nos interesa en el presente trabajo. En concreto, pretendemos demostrar que la gramática codifica diferentes niveles de 
intersubjetividad y que esta diferenciación representa un aspecto constitutivo de la evolución semántica de elementos y construcciones gramaticales. Como objeto de análisis hemos escogido la evolución semántica de la preposición ante y de diversas construcciones gramaticales nucleadas por ella. El itinerario que vamos a seguir es el siguiente: en la próxima sección presentaremos y discutiremos brevemente el concepto fenomenológico de la intersubjetividad, deteniéndonos especialmente en la propuesta de que el complejo conjunto de capacidades que integran este fenómeno puede ser dispuesto en niveles de acuerdo con su complejidad y las fases de su desenvolvimiento (filo- y ontogenético). A continuación, en la sección 3, trazaremos, a grandes rasgos, la evolución semántica de la preposición ante desde su origen latino hasta su uso actual en la lengua española e intentaremos demostrar que una de las características más notorias de dicha evolución es el incremento de la complejidad del tipo de intersubjetividad codificada por la preposición. Finalizaremos el artículo con algunas reflexiones de carácter más general acerca de la relevancia de la intersubjetividad como categoría codificada gramaticalmente.

\section{NIVELES DE INTERSUBJETIVIDAD Y LA CAPACIDAD META-REPRESENTACIONAL DEL LENGUAJE}

En la Fenomenología y, especialmente, en Husserl ${ }^{1}$, la intersubjetividad es la cualidad esencial de la existencia del ser humano, constitutiva tanto del propio sujeto como de la noción de un mundo objetivo (Depraz, 2001: 169).

Para explicar cómo se constituye la intersubjetividad en el propio sujeto (y no en la comunicación, pues es una condición para su posibilidad), Husserl sitúa en el centro de su reflexión filosófica la esfera primordial del Yo. Esta esfera, caracterizada por la conciencia del propio cuerpo (entendido como Leib, es decir, como cuerpo vivo y vivido por mí), representa la esfera de lo mío propio y todo lo que la traspasa algo extraño al Yo. A partir de ahí, Husserl intenta, en varios pasos, describir cómo se crea, en el sujeto, la referencia a todo lo que es extraño al Yo, al "no-yo" (a lo "no-egóico", "das Ich-Fremde"). En un primer paso, percibo la presencia de otro cuerpo que se parece al mío. Esta semejanza me lleva a una asociación por emparejamiento ("Paarungsassoziation") entre mi cuerpo y el otro cuerpo percibido, lo que a su vez me motiva a atribuirle al cuerpo percibido la cualidad de cuerpo vivo ("Leib”), así como la posesión de una conciencia sobre su cuerpo vivo ("Leibbewußtsein"), a imagen y semejanza de mí mismo. Y, finalmente, considero que este proceso de asociación por emparejamiento es recíproco, es decir, atribuyo al otro la facultad de hacer lo mismo al percibir mi cuerpo:

\footnotetext{
${ }^{1}$ Para la exposición del concepto de intersubjetividad en Husserl hemos tomado como texto base Husserl (2002).
} 
Leicht verständlich ist auch die Art, wie eine solche Fremdappräsentation im beständigen Fortgang der wirksamen Assoziation immer neue appräsentative Gehalte liefert (...). Den ersten bestimmten Gehalt mu $\beta$ offenbar das Verstehen der Leiblichkeit des Anderen und seines spezifisch leiblichen Gehaltens bilden: das Verstehen der Glieder als tastend oder auch stoßend fungierende Hände, als gehend fungierende Füße, als sehend fungierende Augen usw (...). In weiterer Folge kommt es begreiflicherweise zur Einfühlung von bestimmten Gehalten der höheren psychischen Sphäre. Auch sie indizieren sich leiblich und im außenweltlichen Gehaben der Leiblichkeit, z.B. als äußeres Gehaben des Zornigen, des Fröhlichen, etc. - wohl verständlich con meinem eigenen Gehaben her unter ähnlichen Umständen² (Husserl, 2002: 198-199).

En síntesis, la intersubjetividad en Husserl hace referencia a la capacidad esencial del sujeto de ponerse en el lugar del otro, de cambiar de lugar ("Platzswechseln"). Al hacerlo, extiendo al otro mis características y capacidades. Entre ellas se encuentran todas las competencias psicológicas y cognitivas superiores: el raciocinio lógico, el establecimiento de relaciones causales entre entidades percibidas, etc.

Dicha capacidad constituye una condición para la posibilidad del uso del lenguaje, y esto en un doble sentido. Por un lado, desde una perspectiva evolutiva (tanto filo- como ontogenéticamente), el desarrollo del lenguaje, como un sistema simbólico convencional, presupone el dominio de lo que se denomina un conocimiento de tercer orden, esto es, yo sé que tú sabes que yo sé (cf. Zlatev, 2008: 232-237; Itkonen, 2008: 288-290). Esto, a su vez, significa reconocer al Otro como agente mental, dotado de las mismas capacidades que yo poseo (incluso de la capacidad de reconocer lo mismo en mí).

Por otro lado -y este es el aspecto que más nos interesa en el presente trabajo-, la intersubjetividad es un elemento constitutivo esencial de la base sobre la que erguimos nuestra conceptuación lingüística del mundo. Esto significa que la conceptuación del mundo mediante el lenguaje no se lleva a cabo por un sujeto aislado, sino desde la perspectiva de un sujeto trascendente que incluye, como algo esencial en su visión y experiencia del mundo, siempre ya la presencia de la pers-

2 "Puede también entenderse fácilmente el modo como semejante apresentación de lo otro, en el constante progreso de la asociación activa, va proporcionando siempre nuevos contenidos apresentativos; o sea, cómo trae a conocimiento definido los contenidos cambiantes del otro ego (...). Tiene, evidentemente, que constituir en el primer contenido definido la comprensión de la corporalidad viva del otro y de su comportamiento específicamente corporal: la comprensión de los miembros como manos que actúan palpando o empujando, como pies que actúan andando, como ojos que actúan viendo, etcétera (...). Más adelante se llega, en forma comprensible, a la "endopatía" de contenidos definidos de la "esfera psíquica superior". También éstos dan indicio corporal de sí, y lo hacen en el comportamiento exterior y mundano de la corporalidad, por ejemplo: como conducta exterior de quien está encolerizado, de quien está alegre, etcétera; conducta que se puede comprender bien desde mi propio comportamiento en circunstancias afines" (Husserl, 2002, trad. esp. 2005: 168-169). 
pectiva del Otro. En resumidas cuentas: el sujeto-conceptuador es, en sí mismo, siempre ya un sujeto diádico.

De ello se desprende, necesariamente, que la intersubjetividad forma parte de la estructura semántica del lenguaje, en general, y de la gramática, en particular. Esto no significa, no obstante, que su presencia e importancia sean siempre fácilmente identificables, puesto que, en muchos casos, la intersubjetividad no es nombrada directamente y permanece, más bien, como un elemento implícito de la conceptuación. Es lo que ocurre, por ejemplo, con los conceptos de tiempo y espacio codificados por la gramática. Cuando, al proferir un enunciado como $V i$ a Juan, ubico temporalmente el evento nombrado con relación al momento presente, el momento presente en cuestión no me pertenece exclusivamente a mí, sino que incluyo, tácitamente, en él también a mi interlocutor. La temporalidad generada es, por tanto, diádica, intersubjetiva, aunque no haya ningún elemento lingüístico que explicite esta cualidad.

Existen, por el contrario, muchas otras construcciones gramaticales que evocan la intersubjetividad de una forma más explícita, presentándola, claramente, como una faceta integrante de la estructura conceptual que codifican:

a. Yo sé muy bien lo que tú piensas al respecto.

El predicado de pensamiento (sé) y su complemento oracional (que tú piensas) evocan en (1) explícitamente una determinada configuración de intersubjetividad, en la que el hablante concibe a su interlocutor como sujeto mental que piensa algo respecto de algo. En este caso, la configuración de intersubjetividad constituye un aspecto focalizado de la semántica de dicha construcción gramatical. Como señala Zlatev (2008: 236), para que enunciados como en (1) tengan sentido, hemos de ser capaces de pensar la intersubjetividad que codifican. Es especialmente en estos casos en los que la capacidad meta-representacional del lenguaje aflora de forma más nítida: el lenguaje nos identifica como seres intersubjetivos.

Como anticipamos en la introducción, nuestro principal propósito en el presente trabajo es demostrar que la gramática no codifica la intersubjetividad de una forma holística, indiferenciada, sino que es sensible a la codificación de capacidades específicas que integran ese complejo fenómeno que llamamos intersubjetividad. Además, en el caso particular de ante, es posible constatar un incremento del grado de complejidad de la capacidad interactiva codificada en cada fase de la evolución semántica de esta preposición.

La idea de que la intersubjetividad es un fenómeno complejo constituido por un conjunto de capacidades individuales está ya presente de forma clara en el propio Husserl (Costelo, 2012). Así, la asociación por emparejamiento -proceso 
que, como mencionamos, es imprescindible para la emergencia de la intersubjetividad- afecta, en un primer momento, al cuerpo vivo-y-vivido (Leib). Al percibir un cuerpo que se asemeja al mío establezco una relación entre ambos y atribuyo al cuerpo que se me presenta todas las cualidades que identifico en el mío, incluyendo la capacidad de poseer un Leibbewußtsein, una conciencia sobre el cuerpo vivo-y-vivido. Y esto, obviamente, en una absoluta reciprocidad: tú haces lo mismo conmigo. El resultado es la conciencia de que compartimos el mismo esquema corporal, esto es, nuestros cuerpos vivos-y-vividos experimentan una semejanza fundamental en el funcionamiento de sus (nuestras) capacidades táctiles, auditivas, visuales, propioceptivas, kinestésicas, etc. (Depraz, 2001: 172-173; Huelva Unternbäumen 2013a: 8-19).

A partir de ese momento, extiendo al otro también todas mis características y competencias cognitivas y psicológicas superiores: afectividad, raciocinio lógico, agentividad, volicionalidad, etc. Este proceso de expansión comienza por un cambio de lugar (Platzwechseln). Imaginativamente me pongo en tu lugar y tú en el mío. Me imagino viendo lo que tú ves, viéndolo desde tu perspectiva o, mejor dicho, desde la que yo tendría si ocupase tu lugar (y viceversa). Este cambio corporal-kinestésico imaginado abre el camino al cambio de estados psicológicos. Me puedo imaginar lo que pensaría o sentiría si ocupase tu lugar y viese lo que tú ves, puedo imaginarme lo que decidiría hacer o lo que no haría en ningún caso. Y, por último, consigo reconstruir tu raciocinio y tus procesos afectivos ante un estado de cosas o un hecho y entender (o entender mal) lo que dices y haces (Depraz, 2001: 173-174).

En síntesis, la intersubjetividad en Husserl se inicia con aspectos predominantemente corporales (el compartimiento del mismo esquema corporal) y se va extendiendo desde ahí a aspectos cada vez más complejos y abstractos pertenecientes a nuestro mundo cognitivo y afectivo. Con ello, el cambio de lugar no se restringe al dominio físico, espacio-temporal, sino que se adentra al mundo de lo que pensamos y sentimos.

Esta idea de una jerarquización de las capacidades intersubjetivas ha sido adoptada también por los estudios del desarrollo de inspiración fenomenológica. Especialmente Trevarthen aporta, en numerosos estudios (cf. por ej. 1979, 1980, 1998), una elaboración detallada, así como una extensa verificación experimental de dicha idea. De un modo general, este autor propone diferenciar entre una intersubjetividad primaria y una secundaria. La intersubjetividad primaria consiste en un conjunto de capacidades sensorio-motoras (innatas o desarrolladas en los primeros meses de vida) que se aplican en contextos interactivos y que nos ayudan a entender lo que las otras personas piensan y sienten a partir de la percepción de sus movimientos, gestos, expresiones faciales, dirección de las miradas, cambios en la entonación y de las acciones que ejecutan en el contexto de interacciones comunicativas. Así, por ejemplo, a partir de los dos meses de vida los niños son capaces 
de seguir la mirada de otra persona, de ver que está mirando en una dirección concreta y de percibir lo que está observando, de tal forma que ponen de relieve la intención de la persona en cuestión (Baron-Cohen, 1995).

La intersubjetividad secundaria se inicia con el desarrollo de la atención conjunta a partir de los 9 meses de edad. A partir de esta edad, los niños adquieren la capacidad de dirigir su atención al mismo objeto al que otra persona dirige la suya. Esta capacidad de compartir el mismo foco de atención es una condición esencial para la posibilidad de adquirir y participar en la construcción de un mundo de la vida común (de co-construir una Lebenswelt compartida) (Gallagher, 2012: 197-198), puesto que con ella los niños son capaces de aprender cómo los otros observan y usan los objetos del mundo y cómo se establecen y desarrollan las relaciones interpersonales mediante el uso de objetos. A medida que ellos mismos comienzan a participar en este tipo de acciones, se van incorporando al proceso de co-construcción del sentido del mundo, a un permanente proceso de creación participativa de sentido (De Jaegher \& Di Paolo, 2007), lo que, a su vez, les permite ir mejorando cada vez más su capacidad de interpretar el comportamiento, las prácticas, los motivos, las razones, etc., de los otros en contextos específicos (Gallagher, 2012: 197). O, reformulado en una terminología más fenomenológica: la capacidad de ponerse cognitiva y afectivamente en el lugar del otro.

Por último, cabe destacar que también los estudios sobre la evolución humana proponen, de un modo general, un aumento paulatino de la complejidad en el proceso del desarrollo filogenético de las capacidades intersubjetivas (cf. p. ej. Tomasello, 1999; Tomasello, Carpenter, Call, Behne \& Moll, 2005; Tomasello \& Call, 2006; Pika, 2008; Hutto, 2008; Zlatev, 2008). A continuación reproducimos un cuadro de Zlatev (2008: 237) que representa los principales estadios o niveles que integran este proceso evolutivo:

Tabla I. Niveles de intersubjetividad, de mímesis y tipos de mentalidad (según Zlatev, 2008: 237).

\begin{tabular}{|c|c|c|}
\hline Nivel de mímesis & Capacidades intersubjetivas & Tipo de pensamiento \\
\hline 1. Protomímesis & $\begin{array}{l}\text { - } \text { Imitación neonatal } \\
\text { - Empatía simple } \\
\text { - }\end{array}$ & $\begin{array}{l}\text { De primer orden (sin } \\
\text { diferenciación entre Yo y } \\
\text { Otro) }\end{array}$ \\
\hline 2. Mímesis diádica & $\begin{array}{ll}\text { - } & \text { Empatía cognitiva } \\
\text { - } & \text { Atención compartida } \\
\text { - } & \text { Comprensión de las } \\
& \text { intenciones del otro en } \\
\text { contextos de competición }\end{array}$ & $\begin{array}{l}\text { De segundo orden } \\
\text { (comprensión del Otro } \\
\text { a través de proyecciones; } \\
\text { identificación, pero } \\
\text { diferenciación) }\end{array}$ \\
\hline
\end{tabular}


RLA. Revista de Lingüística Teórica y Aplicada, 52 (2), II Sem. 2014

Continuación Tabla I.

\begin{tabular}{|l|l|l|}
\hline 3. Mímesis triádica & $\begin{array}{l}- \text { Atención conjunta } \\
-\end{array}$ & Posesión y comprensión de \\
intenciones comunicativas & & \\
4. Post-mímesis & $-\begin{array}{l}\text { Convenciones semánticas } \\
\text { Comprensión de (falsas) } \\
\text { creencias }\end{array}$ & De tercer orden \\
\hline
\end{tabular}

Zlatev propone una coevolución -caracterizada por un paulatino aumento de la complejidad- entre el nivel de mímesis, el tipo de pensamiento y el desarrollo de capacidades intersubjetivas. El proceso de evolución de la mímesis corporal (definida como el uso del cuerpo para fines comunicativos y representativos, Zlatev, 2008: 215) se inicia con la protomímesis, estadio en el cual se lleva a cabo ya una imitación de las acciones corporales del otro sin existir, no obstante, una conciencia de la diferencia entre mi cuerpo y el cuerpo del Otro. Corresponde a este estadio el tipo de pensamiento de primer orden, es decir, todavía no se ha adquirido la capacidad de tener un pensamiento sobre otro pensamiento, ya sea propio o perteneciente al Otro. Las capacidades interactivas típicas de esta fase evolutiva (filo- y ontegenética) son la empatía afectiva simple y la atención mutua (al cuerpo del Otro, no a un objeto). La mímesis diádica, por su parte, ya supone el uso consciente del propio cuerpo para imitar movimientos de objetos o de acciones del Otro. Significa, además, la capacidad de identificarse y, al mismo tiempo, de diferenciarse del Otro. La situación que describe de forma más clara este estadio es la que se constituye en momentos de atención compartida: te observo y veo que tú diriges tu atención a un objeto y te imito haciendo lo propio. Esta situación requiere la posibilidad de poseer una empatía cognitiva, esto es, la capacidad de ponerse mentalmente en el lugar del Otro y de reconstruir lo que él está pensando: te veo observando un determinado objeto y pienso que lo deseas tener. Consecuentemente, tenemos aquí ya un pensamiento de segundo orden. El sujeto es capaz de dirigir su pensamiento sobre otro pensamiento: pienso que tú piensas $\mathrm{X}$, creo que haces $\mathrm{X}$ por tal o cual motivo o razón, me parece que estás en tal o cual estado anímico, etc. Las situaciones de atención compartida son además, según Zlatev (2008: 226-232), el origen más probable de uno de los cambios más significativos en la evolución del homínido al ser humano, a saber, el surgimiento de la mímesis triádica y, con ella, del pensamiento de tercer orden y las capacidades intersubjetivas superiores: te observo y veo que tú diriges tu atención a un objeto y me percato de que tienes la intención de que yo también dirija mi atención al mismo objeto (atención conjunta). Surge así una situación comunicativa simple, 
basada en la posesión y comprensión de una intención comunicativa. Corresponde a esta situación un pensamiento de tercer orden: pienso que tú piensas que yo pienso $\mathrm{X}$ ( $\mathrm{y}$ viceversa, claro). $\mathrm{O}$, aplicado a nuestra situación concreta, yo pienso que tú tienes la intención de que yo observe el objeto en cuestión. Y, finalmente, lo que diferencia la mímesis de tercer orden de los estadios posmiméticos es el uso de signos totalmente convencionales interrelacionados en un sistema, es decir, el uso del lenguaje para fines comunicativos (Zlatev, 2008: 232-236). En este estadio, mediante la utilización del lenguaje, adquirimos lo que hemos denominado capacidad meta-representacional: la posibilidad de utilizar el lenguaje para conceptuar y expresar las diversas capacidades intersubjetivas que configuran nuestra condición humana.

Conviene precisar, antes de concluir nuestra caracterización de la jerarquización de la intersubjetividad, que aunque tanto los estudios sobre el desarrollo ontogenético como los dedicados a la evolución filogenética postulen una distribución de las capacidades intersubjetivas en diferentes niveles o estadios, esto no supone que con el paso de un estadio al otro perdamos las capacidades intersubjetivas del estadio anterior. Los procesos de desarrollo y evolución tienen más bien un carácter acumulativo, esto es, adquirimos nuevas capacidades que se suman a las que ya poseíamos (Zlatev, 2008: 219; Gallagher, 2012: 198-199). Esto supone que la capacidad meta-representacional del lenguaje puede, en principio, codificar y expresar cualquiera de las capacidades intersubjetivas que hemos ido describiendo a lo largo de esta sección. Veamos, para finalizar, algunos ejemplos.

Como es ampliamente conocido, el cuerpo humano constituye, en la mayoría de las lenguas del mundo, el punto de partida (el llamado dominio fuente) para la conceptuación lingüística de relaciones espaciales (cf. p. ej. Heine, 1995, 1997; Heine, Claudi \& Hünemeyer, 1991; Heine \& Kuteva, 2002). Los conceptos de referenciación espacial codificados gramaticalmente (especialmente por preposiciones) se fundamentan sobre el conocimiento que tenemos sobre nuestro cuerpo. Que la mayoría de las lenguas (de culturas y geografías diferentes) procedan del mismo modo en la conceptualización de relaciones espaciales sólo es explicable porque todos los seres humanos compartimos el mismo esquema corporal y porque todos sabemos que lo compartimos (Huelva Unternbäumen, 2003a: 8-19). La posibilidad, por ejemplo, de derivar el concepto de la anterioridad espacial de la frente se debe, en primer lugar, al hecho de que compartimos el mismo esquema corporal, caracterizado entre otras cosas por una frontalidad perceptiva que sitúa nuestros órganos perceptivos más importantes en la parte frontal de la cabeza, de tal modo que nos hemos de posicionar 'frontalmente' al objeto que queremos percibir. En segundo lugar, nos hemos de percatar de que compartimos esta característica de nuestro esquema corporal. Esto es, hemos de ser capaces de configurar una asociación por emparejamiento con respecto a la frontalidad perceptiva de nuestro esquema corporal. La conciencia sobre el valor intersubjetivo 
de este aspecto del esquema corporal garantiza que los conceptos espaciales que derivemos de él puedan, a su vez, adquirir también valor intersubjetivo, requisito indispensable para cualquier concepto a ser codificado por la lengua. La intersubjetividad del esquema corporal hace con que sea el punto de partida ideal (el dominio fuente ideal) para la creación de otros conceptos intersubjetivos. Y, finalmente, la posibilidad de derivar un concepto de relación temporal de una parte del cuerpo requiere que seamos capaces de ponernos imaginativamente en el lugar del otro (Platzwechseln). Cuando profieres enunciados como "estoy frente a ti" o "estoy sentado frente al mar" tengo que ser capaz, para entenderlos debidamente, de ponerme en tu lugar y verme a mí mismo como tú me ves (o como yo me vería si ocupase tu lugar y me observase a mí mismo) y a observar el mar como tú lo observas (o como yo lo observaría si estuviese en tu lugar). En síntesis, debemos concluir que forman parte de la estructura conceptual de la preposición frente a (i) la frontalidad perceptiva como uno de los aspectos centrales de nuestro esquema corporal, (ii) el conocimiento sobre el carácter intersubjetivo de este aspecto y (iii) la capacidad evocada por el propio uso de la preposición de adoptar la perspectiva del otro e incluirla en nuestras percepción del mundo.

¿Qué lugar en la jerarquía de la intersubjetividad ocuparía esta estructura conceptual? Uno no muy elevado, que incluye, fundamentalmente, el compartimiento del mismo esquema corporal y de una cierta atención compartida. Decimos 'cierta' porque se restringe a la dimensión perceptiva sin adentrarse en aspectos de una empatía cognitiva o afectiva. Para entender tu enunciado "estoy sentado frente al mar" he de ser capaz de situarme imaginativamente en tu lugar y de intuir lo que tú ves. La estructura conceptual del enunciado no nos dice nada respecto a lo que sientes o piensas al observar el mar, ni a los motivos que te llevaron a hacerlo, ni a las acciones o intenciones que tal observación eventualmente pueda causar en ti. Se trata, pues, más que de una intersubjetividad plena, de una intercorporeidad, de un compartimiento del mismo esquema corporal (Huelva Unternbäumen, 2013a: 8-19).

Otros elementos y construcciones gramaticales codifican una intersubjetividad más compleja. Es lo que ocurre, por ejemplo, con las construcciones ditransitivas. El concepto de la Transferencia de Objeto, que corresponde a la estructura semántica prototípica de dichas construcciones, constituye el caso paradigmático de la acción conjunta, en el sentido explanado más arriba. En una situación de transferencia de objeto (material), los dos agentes involucrados (el que da el objeto y el que lo recibe) coordinan intencionalmente sus acciones siguiendo un conjunto de patrones de comportamiento cooperativos necesarios para alcanzar la meta compartida: se sitúan uno frente al otro a una determinada distancia, prestan mutuamente atención a los movimientos del otro y, conjuntamente, al objeto a ser transferido, coordinan los movimientos de los brazos y las manos de tal forma que el objeto no se caiga, etc. Cada uno de los dos agentes está consciente de 
que él mismo y el otro están atentos al mismo objeto y al mismo evento y que interactúan, por lo tanto, en una relación triádica (agente-agente-objeto/evento) (Gallagher, 2013: 226-227).

La configuración de intersubjetividad que tenemos en este caso es bastante más compleja que la que hemos constatado para la preposición frente a. Esta mayor complejidad se pone de manifiesto ya al nivel de lo que hemos denominado intercorporeidad. La construcción ditransitiva codifica una interacción entre dos agentes que sólo puede iniciarse y llevarse a cabo porque ambos comparten el mismo esquema corporal (especialmente, la misma frontalidad sensorio-perceptiva y motora) y porque saben recíprocamente que lo comparten. Además es importante observar que el uso de dicha construcción en un enunciado como por ejemplo Juan le da las llaves a su prima supone, implícitamente, que el hablante que profiere tal enunciado también posee el mismo esquema corporal que Juan y su prima. Esto es, el hablante, tiene que ser capaz -y lo es- de ponerse en el lugar tanto del agente que transmite el objeto como del que lo recibe. Tenemos, pues, una intercorporeidad que se establece, como mínimo, entre tres sujetos: los agentes involucrados en la transferencia y el propio hablante que conceptúa la situación observada mediante el uso de la construcción ditransitiva. El aumento más significativo de la complejidad se debe, no obstante, al hecho de que la construcción ditransitiva codifica un pensamiento de tercer orden y, por lo tanto, capacidades intersubjetivas superiores: cuando doy un objeto a alguien pienso que quien lo va a recibir piensa que yo tengo la intención de dárselo. Del mismo modo, cuando recibo un objeto de alguien pienso que quien me lo da piensa que yo quiero recibirlo. Tenemos, pues, la codificación de una situación de "intención compartida" (Gallagher, 2013: 226), que supone que cada uno de los dos involucrados comprende las intenciones del otro y las identifica como compatibles con las suyas propias, en el sentido de aspirar a una meta común.

Acabamos esta sección con la formulación de la hipótesis -a la que parece que nos facultan los ejemplos analizados- de que la capacidad meta-representacional del lenguaje puede manifestarse de forma distinta en diferentes elementos y construcciones gramaticales. Dicho de un modo más concreto: el potencial de codificación de la gramática nos permite distinguir entre diferentes tipos y grados de complejidad de la intersubjetividad. Esta diferenciación se manifiesta también en la evolución diacrónica de un mismo elemento gramatical, como veremos en la próxima sección.

\section{ANTE: DEL ESPACIO A LA CAUSACIÓN}

En latín, ante formaba parte del sistema binario de preposiciones relacionales (super-sub, supra-infra, ante-post, intra-extra, etc., cf. García-Miguel, 2006: 1286- 
1288) y designaba anterioridad, tanto en el dominio espacial como en el temporal (Meyer-Lübke, 1890-1906: III, 516; Penny, 1993: 221). Es difícil verificar si ya en esta lengua existían casos de lecturas causales/causativas (u otro de los valores semánticos actuales de esta preposición) o si -lo que parece más probable- el surgimiento de la semántica causal de ante es concomitante a la extensión del uso de preposiciones compuestas. Así, Penny (1993: 221) señala que ante fue paulatinamente desplazado por antes de (con la "s" que marca su origen adverbial) en el dominio de la anterioridad temporal. De forma análoga, se produjo también un desplazamiento gradual en el dominio locativo, en el que ante se enfrentó con derivados como desante ( $<$ de ex ante), enante ( $<$ in ante) y, especialmente, denante $(<$ de in ante), del que con el tiempo derivó el actual delante de (Meyer-Lübke. 1890-1906: III, 164; Penny, 1993: 221; García-Miguel, 2006: 1296-1303).

Este proceso de substitución parece haberse llevado a cabo de forma lenta y gradual, atravesando un largo periodo caracterizado por una coexistencia del uso de ante y de sus derivados complejos, periodo que, de hecho, se extiende hasta los días de hoy. Valgan aquí algunos ejemplos de este uso concomitante a través de los siglos ${ }^{3}$.

a. Esto es por fuero de vn omne que muestra sennal de juez delante dos vezinos derechos \& non viene fazer derecho ante el alcalle deue pechar çinco sueldos (CORDE. Anónimo: 1200-1300).

b. Palabras d' Amos, que fue de los brusceros de Tecoa, que prophetizo sobre Israel, en dias de Osias rey de Judea, en dias de Jheroboam, fijo de Joas, rey [f. 71v] de Israel, .ii. annos ante de la tempestad (CORDE. Almerich: 1200).

c. E después que ofrescieron, salieron fuera ante la iglesia, que avía una plaça grande e llana, en la qual vieron un padrón quadrado(...) (CORDE. Anónimo: 1400).

d. La sentencia destos versos es ésta: quando pasares delante de la imagen de santa María non se te oluide el Aue María, que aquel que en este mundo la saludare con el Aue María en el otro mundo será saludado (CORDE. Anónimo: 1400-1500).

e. Estando assí airado no havía cavallero que se osase parar delante dél para le dezir cosa alguna, y assí estovo toda aquella noche. Y otro día por la mañana todos los cavalleros fueron ante las puertas del palacio del rey y el rey Sornaguer con ellos,

${ }^{3}$ Los ejemplos pertenecen a Huelva Unternbäumen (2013b). 
Niveles de intersubjetividad y conceptualización gramatical: El caso del cambio semántico de la preposición ante / E. HuELVA

mas no porque lo conosciessen, que assí estava entre ellos como si fuera otro cavallero (CORDE. Anónimo: 1500).

El primer ejemplo registrado en el CORDE, al que podemos atribuirle esta dimensión, data de 1200 :

He ellos que yvan fuyendo ante los de Israel, echo Nuestro Sennor piedras grandes del cielo a la tierra e $\mathrm{m}[\mathrm{u}]$ rieron dent muchos; mas fueron los que murieron de piedras que los que murieron a espada (CORDE. Almerich: 1200).

El autor de (3) no expresa una mera relación espacial entre "ellos" y "los de Israel", sino la razón por la cual "ellos" huyeron. Es decir, el autor se coloca en el lugar del otro ("ellos") que percibe la presencia de los de Israel y concluye que esta presencia es un motivo para huir.

Este paulatino desplazamiento de ante por antes y por delante de, en el dominio temporal y espacial respectivamente, parece ir acompañado de un proceso de especialización de la primera en casos en los que no tenemos tan solo un sujeto-conceptuador que conceptúa ('objetivamente') una relación espacio-temporal externa entre objetos o eventos, sino en los que también y sobre todo se pretende poner de manifiesto la existencia de una configuración de intersubjetividad: el compartimiento entre dos o más sujetos-conceptuadores de la conceptuación de una determinada relación (así como de las capacidades y operaciones necesarias para llevarla a cabo). Esta dimensión intersubjetiva es común a todos los valores semánticos actuales de ante y la hemos constatado en más del 90\% de los 3.045 enunciados analizados, correspondientes a todas las ocurrencias del año 2004 del CREA (Huelva Unternbäumen, 2013b).

Centrémonos primero en el desarrollo de la semántica causativa. En el proceso de evolución de una semántica exclusivamente espacial a una causativa podemos identificar tres estadios diferentes, que se caracterizan claramente por un incremento del grado de complejidad de la intersubjetividad codificada, en el sentido que hemos expuesto en la sección anterior.

El punto de partida lo constituye el uso de ante con valor locativo, esto es, para expresar una relación espacial entre dos objetos. Como dijimos, este valor semántico es expresado en el español actual mayoritariamente por la preposición delante $d e$, que substituyó paulatinamente en esta función a ante. De las 3.045 ocurrencias de ante analizadas en Huelva Unternbäumen (2013b), menos del 5\% expresa tan solo una relación de anterioridad física, observada por el hablante/conceptuador. Los ejemplos en (4) pertenecen a este tipo: 
(4)

a. Las colas de ayer ante los colegios electorales de los Estados Unidos ratifican una movilización sin precedentes de nuevos electores.

b. Un numeroso grupo de iraquís rezan ante la puerta de la mezquita del imán Alí.

La expresión de una anterioridad meramente física exige, obviamente, que el elemento nominal después de ante contenga un sustantivo concreto que denote un objeto en el dominio espacio-temporal (los colegios electorales en 4 a y la puerta en 4b). Sin embargo, incluso en muchos casos en los que se cumple esta exigencia no se puede negar la existencia e incluso la predominancia de una clara dimensión intersubjetiva en la relación expresada por ante. Observemos el contraste entre después de y ante en (5):

(a) Cuando me siento delante de la tele para ver un capítulo de Buffy es algo así como estar ante un buen libro de cómo escribir guiones.

La preposición compuesta delante de expresa la relación espacial entre un objeto (tele) y el local en el que se ejecuta una acción (sentarse). Por el contrario, estar ante un buen libro no designa sólo y ni siquiera predominantemente una relación espacial entre un individuo y un objeto, sino, y ante todo, una relación cognitiva. El libro no es aquí tan sólo -ni siquiera predominantemente- un objeto del mundo físico, sino un objeto de conciencia, cuyo contenido es percibido, conceptuado y evaluado por el sujeto. Para facilitar el análisis, consideremos también el siguiente ejemplo construido:

(6)

Se desmayó delante del féretro de su marido.

Consideremos, para nuestro análisis, al hablante como primer sujeto conceptuador $\left(\mathrm{SC}_{1}\right)$, al oyente o lector como segundo sujeto conceptuador $\left(\mathrm{SC}_{2}\right)$ y a las personas nombradas en el enunciado como tercero, cuarto y así sucesivamente $\left(\mathrm{SC}_{3}, \mathrm{SC}_{4}, \mathrm{SC}_{\mathrm{n}}\right)$. Lo primero que llama la atención, al analizar el enunciado en (6), es que la preposición delante de no codifica necesariamente que $\mathrm{SC}_{3}$ percibe el objeto nombrado (féretro). Quien establece la relación espacial entre el objeto (féretro) y el evento (desmayarse) es $\mathrm{SC}_{1}$, en la calidad de observador externo. Consecuentemente, no existe intersubjetividad entre $\mathrm{SC}_{1}$ y $\mathrm{SC}_{3}$ (quien sufre el 
desmayo) con respecto a la percepción de la relación espacial en cuestión. Lo que sí existe, obviamente, es una intersubjetividad -más tácita que explícita- entre $\mathrm{SC}_{1}$ y $\mathrm{SC}_{2}$, que reside en el hecho de que el hablante presupone que si el oyente estuviese en su lugar observaría y percibiría la situación del mismo modo que él. Es lo que podríamos llamar una configuración de intersubjetividad básica, pues es inherente a todo acto de enunciación (Huelva Unternbäumen, 2013a: 21-22). Finalmente, cabe señalar que (6), así como cualquier otra relación espacial que se establezca entre un objeto y el cuerpo de un ser humano (Leib) o entre dos cuerpos, codifica una intercorporeidad, un compartimiento del mismo esquema corporal, entre $\mathrm{SC}_{1}$ y $\mathrm{SC}_{3}, \mathrm{SC}_{4}, \mathrm{SC}_{\mathrm{n}}$

Veamos ahora nuevamente los ejemplos en (4), que repetimos a continuación para facilitar el análisis:

a. Las colas de ayer ante los colegios electorales de los Estados Unidos ratifican una movilización sin precedentes de nuevos electores.

b. Un numeroso grupo de iraquís rezan ante la puerta de la mezquita del imán Alí.

Los enunciados en (7) denotan una relación espacial como el resultado de una acción (intencional) de posicionamiento, de ubicación. Los votantes se posicionan ante el colegio electoral, formando así colas. Y de forma análoga, los fieles dirigen su atención hacia la puerta de la mezquita para orar. En estos casos, la relación espacial no es tan sólo el producto de la percepción y conceptuación de un observador externo $\left(\mathrm{SC}_{1}\right)$, sino que engloba también la percepción y conceptuación de los individuos mencionados en los enunciados $\left(\mathrm{SC}_{3}, \mathrm{SC}_{4} \ldots \mathrm{SC}_{\mathrm{n}}\right)$. Para que los votantes puedan dirigirse a los colegios electorales tienen primero que ser capaces de percibirlos y de percibirse a sí mismo en una determinada posición relativa al lugar al que pretenden ir. Lo mismo vale para los fieles y su posicionamiento con respecto a la mezquita. Por consiguiente, la preposición ante codifica en estos casos una intersubjetividad perceptiva y cognitiva y no tan sólo el compartimiento del mismo esquema corporal. La conceptuación de la situación en cuestión por parte de $\mathrm{SC}_{1}$ incluye, como elemento esencial, la atribución a $\mathrm{SC}_{3}, \mathrm{SC}_{4} \ldots \mathrm{SC}_{\mathrm{n}}$ de una operación de percepción y autopercepción y de conceptuación y autoconceptuación en el mundo: yo te percibo y concibo como yo mismo me percibiría y concebiría si estuviese en tu lugar (ante los colegios electorales o la puerta de la mezquita).

Esta inclusión de una intersubjetividad perceptiva y cognitiva es el factor determinante que permite el surgimiento de inferencias causativas. Con ello llegamos al segundo estadio en la evolución de la semántica de ante. A él pertenecen ejemplos 
como los que tenemos en (8):

a. Se desmayó ante el féretro de su marido.

b. Un viajero menorquín, que se llamaba José María Cuadrado, que recorrió España en el siglo pasado, bajó Navacerrada y se quedó sorprendido ante lo que vio.

c. Anoche, por ejemplo, no dejaba de sorprenderme ante la casualidad de estar cenando con mis ex-compañeros de ex-curso dos semanas después de lo que ha pasado.

d. Semejante situación atormentó durante más de dos décadas a los físicos de la época, consternados ante su incapacidad de zanjar satisfactoriamente el problema.

En (8a) tenemos una intersubjetividad perceptiva y cognitiva semejante a la que acabamos de describir para los enunciados en (7). El elemento nuevo que surge aquí es, no obstante, que la inclusión del mundo perceptivo y cognitivo de $\mathrm{SC}_{3}, \mathrm{SC}_{4} \ldots \mathrm{SC}_{\mathrm{n}}$, como parte inherente de la conceptuación, abre las puertas para que $\mathrm{SC}_{1}$ pueda incluir aspectos constitutivos de este mundo -a saber, estados y procesos afectivos, psicológicos, procesos de razonamiento, de toma de decisiones, etc. $-\mathrm{y}$ de relacionarlos con la percepción de un objeto. Nace así la posibilidad de hacer inferencias de naturaleza causativa o causal. Así, la percepción del féretro es concebida como la causa del desmayo. A la intersubjetividad de la percepción de una relación espacial se sobrepone la intersubjetividad de una relación causal entre una percepción y la ocurrencia de una reacción psíquico-física. Conviene señalar, además, que el acceso al mundo cognitivo de $\mathrm{SC}_{3}, \mathrm{SC}_{4} \ldots \mathrm{SC}_{\mathrm{n}}$ permite que el objeto percibido pueda dejar de ser algo con un correlato directo en el mundo físico y abarcar de este modo también objetos mentales. Es lo que ocurre, con un orden creciente, en los enunciados (8b, c y d). En síntesis, la intersubjetividad codificada por ante en estos casos abarca el compartimiento de la percepción y conceptuación de un objeto, la empatía de afectos, su procesamiento y la reacción a los mismos (por ejemplo, el desmayo).

El tercer y último estadio de la evolución semántica de ante (espacio > causación) se caracteriza por incorporar la codificación de la intersubjetividad de estados y procesos volicionales e intencionales, que, como sabemos, constituyen aspectos semánticos importantes de la causación (Shibatani, 2002: 11-14; Huelva Unternbäumen, 2013b: 18-22). Observemos los enunciados siguientes: 
(9)

(a) Entonces eran grupos que estaban trabajando en sus distintas localidades y que ante el problema de las elecciones del ochenta y cinco decidieron formar una especie de federación de grupos (...).

(b) De manera que, ante la eventualidad de que eso sea posible, quiero centrar la cuestión de privilegio de mi bloque en dos manifestaciones de dos señores senadores.

Repárese que en los enunciados en (9) entre el objeto mental (u objeto de conciencia) percibido por $\mathrm{SC}_{3}, \mathrm{SC}_{4} \ldots \mathrm{SC}$ ("el problema de las elecciones del ochenta y cinco" y "la eventualidad de que eso sea posible", respectivamente) y la ocurrencia de una reacción (el llamado evento causado) se interpone, como elemento mediador, un proceso de razonamiento de $\mathrm{SC}_{3}, \mathrm{SC}_{4} \ldots \mathrm{SC}_{\mathrm{n}}$. La percepción y el procesamiento del objeto mental son concebidos por $\mathrm{SC}_{1}$ como los desencadenantes de un proceso de razonamiento en $\mathrm{SC}_{3}, \mathrm{SC}_{4} \ldots \mathrm{SC}_{\mathrm{n}}$ que, a su vez, lleva a la toma de una decisión. El evento causado se manifiesta, por tanto, como una (re)acción volitiva, como algo pretendido por el sujeto, hecho que queda de manifiesto notoriamente mediante el uso de los verbos decidir y querer en (9). 'Decidir (o querer) hacer algo' supone formar juicio sobre algo y tomar consciente e intencionalmente una cierta determinación. La intersubjetividad codificada en estos casos es de una complejidad considerable, pues abarca principalmente capacidades y procesos cognitivos superiores. A ellos pertenecen la percepción y conceptuación de un objeto mental, la capacidad de formar un juicio sobre el mismo, esto es, de constatar lo que supone o puede suponer su existencia para el sujeto y para los otros, la identificación y la ponderación de posibilidades de acción y, por último, la toma de una decisión. Obsérvese, además, que la intercorporeidad (el compartimiento del mismo esquema corporal) ha perdido la condición de elemento focalizado de la conceptuación. Es más, ni el objeto de conciencia, ni su relación con el sujetoconceptuador y ni siquiera éste -como poseedor de un esquema corporal concreto- se conceptúan como detentores de un correlato externo directo.

De este modo, la relación causal/causativa codificada por ante no configura lo que tradicionalmente se conoce en la bibliografía como una "causa real" o "causa pura” (Galán Rodríguez, 1999: 3600-3602; Sanders, Sanders \& Sweetser, 2009: 20-21), a saber, la que se establece cuando el sujeto conceptuador percibe en el mundo una relación de causa-efecto entre dos estados de cosas o eventos y la representa "objetivamente" mediante sus enunciados. El uso de ante para esta finalidad es inapropiado, como se puede apreciar en los enunciados siguientes: 
a. Los cristales se rompieron por (por causa de, debido a) la explosión de la bomba.

a'. Los cristales se rompieron ante la explosión de la bomba.

b. El edificio se desmoronó por (por causa de, debido a) un corrimiento de las tierras de la ladera.

b'. El edificio se desmoronó ante un corrimiento de las tierras de la ladera.

En los ejemplos en (10) no se explicita ningún sujeto conceptuador, ni siquiera el propio hablante o autor del texto. Por ello es obviamente imposible construir una intersubjetividad de la relación causal expresada (¿entre qué sujetos se establecería?). La relación causal no puede ser ubicada en un sujeto conceptuador, puesto que no se nombra ninguno. A falta de otro sujeto, resta tan sólo un hablante (implícito) que meramente observa y relata eventos situados en el 'mundo objetivo' que están vinculados causalmente.

El uso de ante en estos casos es inapropiado. Repárese que en (10a' y b') ante produce una ruptura del vínculo causal directo y parece incluso consentir una interpretación local de la relación entre los eventos: el edificio se desmoronó cerca de o delante de, pero no necesariamente por causa de un deslizamiento de tierras.

De una forma más sucinta, podríamos decir que con ante no conceptuamos directamente algo en el mundo, sino que más bien conceptuamos lo que -supuesta o realmente- percibe, siente y conceptúa otro sujeto.

Para obtener un panorama más completo y adecuado de la semántica de esta preposición, conviene señalar que la codificación de un alto grado de intersubjetividad no se restringe al dominio causal/causativo, sino que representa un denominador común de todos los valores semánticos de esta preposición en el español actual, exceptuando aquellos pocos casos en que, como dijimos, todavía permanece la expresión de una relación meramente espacial.Veamos algunos ejemplos:

a. El equipo del candidato oficialista anunció que ayer iba a presentar una demanda ante la Comisión Electoral Central, dadas las "falsificaciones sistemáticas". (Presencia ante una autoridad).

b. Mantenga una actitud abierta ante las sugerencias, cambios e interrupciones. (Presencia ante un objeto de conciencia). 
c. Los discursos sobre la cultura de paz son inútiles ante una realidad que se construye con acciones que siguen formando para la guerra. (Comparación entre objetos de conciencia).

d. EL DIARIO no se arredró ante los ataques que recibió (...). (Relación concesiva entre objetos de conciencia).

(Ejemplos de Huelva Unternbäumen, 2013b).

Independientemente del valor semántico específico de cada caso, el primer sujeto conceptuador $\mathrm{SC}_{1}$ conceptualiza, mediante el uso de ante, una relación que se establece en el espacio mental de otro sujeto conceptuador $\mathrm{SC}_{3}, \mathrm{SC}_{4} \ldots \mathrm{SC}_{\mathrm{n}}$. $\mathrm{La}$ relación en sí se constituye entre dos (o más) objetos de conciencia de $\mathrm{SC}_{3}, \mathrm{SC}_{4} \ldots$ $\mathrm{SC}_{\mathrm{n}}$, con la particularidad de que, en algunas ocasiones, uno de estos objetos de conciencia puede ser el propio sujeto $\mathrm{SC}_{3}, \mathrm{SC}_{4} \ldots \mathrm{SC}_{\mathrm{n}}$.

Analicemos brevemente cada uno de los enunciados de (11). El enunciado en (a) es el que mantiene todavía una cierta referencia al mundo exterior. Sin embargo, hemos de admitir que la 'Comisión Electoral' no es un lugar, una localización en el espacio físico, sino que aparece aquí, ante todo, como un determinado concepto del dominio institucional. La relación codificada por ante se sitúa en espacio mental de $\mathrm{SC}_{3}, \mathrm{SC}_{4} \ldots \mathrm{SC}_{\mathrm{n}}$ (El equipo del candidato oficialista) y se establece entre este sujeto y el concepto (objeto de conciencia) 'Comisión Electoral'. En los otros tres ejemplos, la correlación entre los respectivos objetos de conciencia ('una actitud abierta' y 'sugerencias, cambios e interrupciones', en (a); 'Los discursos sobre la cultura de paz' y 'una realidad', en (b); la actitud de no arredrarse y "los ataques”, en c) y objetos concretos del mundo físico es todavía más tenue. Prepondera con ello, de forma más clara, una relación que emerge en el espacio mental del sujeto conceptuador $\mathrm{SC}_{3}, \mathrm{SC}_{4} \ldots \mathrm{SC}_{\mathrm{n}}$ y que se refiere exclusivamente a entidades internas de dicho espacio. La diferencia entre ellos reside en el valor semántico que adquiere la relación en cada caso: en (b) emerge levemente un valor causativo; en (c) se aprecia un valor comparativo entre los objetos de conciencia relacionados, con prevalencia del objeto introducido por ante; y en (d) se manifiesta un valor concesivo.

En cuanto al grado de intersubjetividad codificado, es -como anticipamos más arriba- notablemente alto en todos estos casos. La estructura conceptual de estos enunciados incluye el compartimiento entre $\mathrm{SC}_{1}$ y $\mathrm{SC}_{3}, \mathrm{SC}_{4} \ldots \mathrm{SC}_{\mathrm{n}}$ de la capacidad de percibir y conceptuar objetos mentales complejos y de establecer diferentes relaciones conceptuales entre ellos.

En la Figura 1 resumimos gráficamente las transformaciones semánticas de la preposición ante que hemos ido comentando a lo largo de la presente sección. 
RLA. Revista de Lingüística Teórica y Aplicada, 52 (2), II Sem. 2014

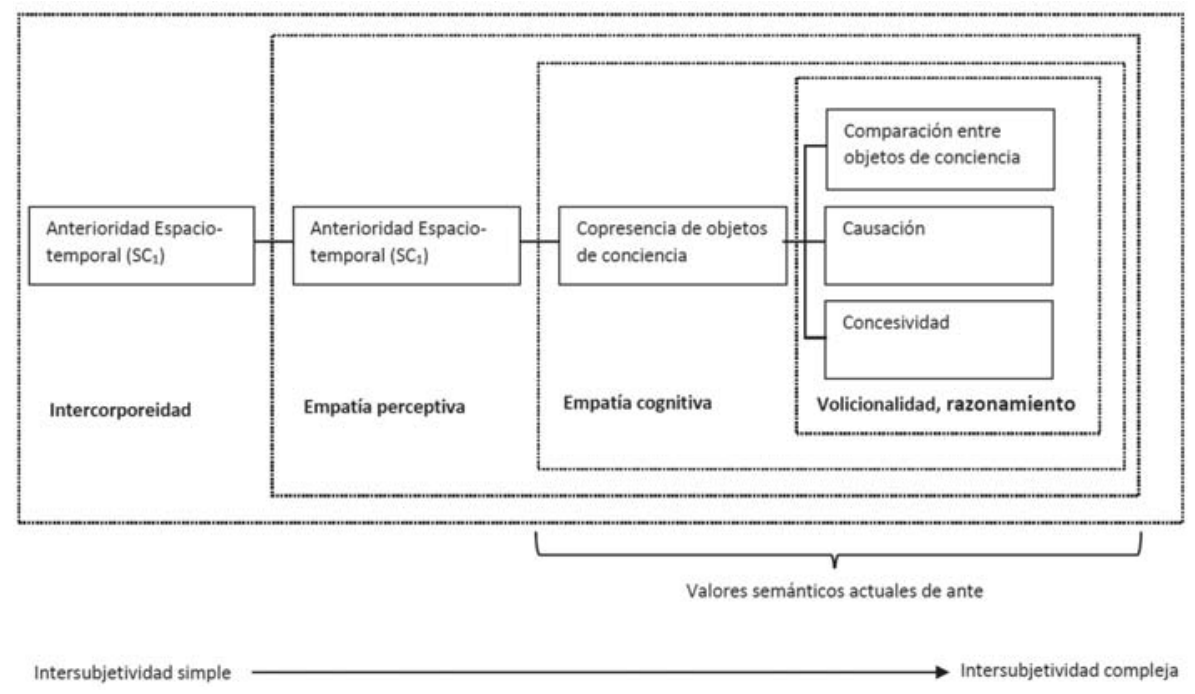

Figura 1. Evolución semántica de ante y niveles de intersubjetividad.

Como se observa en la Figura 1, la evolución de la preposición ante se caracteriza por un proceso de adquisición y pérdida de valores semánticos al que le es inherente un incremento concomitante del grado de intersubjetividad codificado. La intercorporeidad, aspecto constitutivo de la conceptuación 'objetiva' de relaciones espaciales, es sustituida paulatinamente por niveles de una intersubjetividad cada vez más compleja, hasta llegar a la codificación de una empatía de capacidades cognitivas de gran complejidad, como lo son procesos volitivos, de razonamiento y toma de decisiones. Este alto grado de intersubjetividad se manifiesta como componente central de la semántica actual de esta preposición. Su importancia radica especialmente en el hecho de que parece actuar como un factor acotador del potencial de codificación de dicha preposición en los distintos dominios semánticos en que se usa. Como vimos, esto supone, por ejemplo, que ante puede ser usado para conceptuar relaciones espaciales siempre que en la conceptuación el hablante $\left(\mathrm{SC}_{1}\right)$ incluya también al otro $\left(\mathrm{SC}_{3}, \mathrm{SC}_{4} \ldots \mathrm{SC}_{\mathrm{n}}\right)$ como sujeto conceptuador de la relación en cuestión. De forma análoga, demostramos que también en el dominio conceptual de la causalidad/causación el uso de ante es inadecuado para codificar 'causas reales', que carecen de un segundo sujeto conceptuador además del propio hablante. Conviene puntualizar, no obstante, que los niveles más simples de intersubjetividad no desaparecen del todo con la codificación de niveles más complejos. Parece más razonable considerar que se sitúan en un segundo plano, dejando en destaque los niveles más complejos. Así, por ejemplo, 
cuando ponemos de relieve la volicionalidad de una determinada acción ejecutada por un individuo en el contexto de un evento causativo, no por eso dejamos de conceptuarlo como un ser que comparte con nosotros el mismo esquema corporal y capacidades perceptivas semejantes. Esta circunstancia está representada en la Figura 1 por la disposición de los rectángulos punteados.

En definitiva, podemos concluir que una de las principales funciones de la preposición ante en la lengua española actual es la de actuar como un marcador de intersubjetividad en los diversos dominios conceptuales en los que es usada.

\section{CONCLUSIONES}

El análisis que hemos desarrollado en este trabajo nos ha permitido demostrar que la codificación de diferentes niveles de intersubjetividad posee una importancia notable tanto en la evolución semántica de la preposición ante, desde sus orígenes latinos hasta su uso actual en la lengua española, como, desde una perspectiva sincrónica, para acotar debidamente el potencial semántico de dicha preposición en los diferentes dominios conceptuales en los que actúa, delimitando su uso frente al de otros elementos lingüísticos.

Tanto desde la óptica diacrónica como desde la sincrónica, a una diferenciación concreta parece que le hemos de conferir una importancia central, a saber, la que existe entre conceptuar directamente algo del mundo y conceptuar lo que (real o supuestamente) conceptúa el Otro. Esta diferencia es tan fundamental que es razonable suponer que el lenguaje reserve para cada caso formas de expresión propias y que construya y reconstruya posibilidades de conceptuar y expresar este contraste esencial en el proceso de evolución lingüística. Es lo que hemos constatado con respecto al contraste delante de versus ante o al uso de ante en el dominio conceptual de la causalidad/causación frente al de otros elementos lingüísticos.

Finalmente, conviene puntualizar que la diferenciación de niveles de intersubjetividad propuesta por la literatura filosófica y de áreas afines puede coincidir en mayor o menor grado con la codificada por la gramática de las lenguas naturales. Es, obviamente, muy prematuro -y por lo tanto sería absolutamente desacertado- proponer algún tipo de generalización a este respecto. Resta, no obstante, constatar, como punto de partida para futuras investigaciones, que en el caso aquí analizado dicha coincidencia debe ser considerada alta.

\section{REFERENCIAS}

Baron-Cohen, Simon. (1995). Mindblindness: An essay on autism and theory of mind. Massachusetts: MIT Press. 
Costelo, Peter. (2012). Layers in Husserl's phenomenology. On meaning and intersubjectivity. Toronto: University of Toronto Press.

De Jaegher, Hanne \& Di Paolo, Ezequiel. (2007). Participatory sense-making: an enactive approach to social cognition. Phenomenology and the Cognitive Sciences, 6, 485-507.

Depraz, Natalie. (2001). The Husserlian theory os intersubjectivity as alterology: Emergent theories and wisdom traditions in the light of genetic phenomenology. En Thompson, Evan (ed.), Between Ourselves. Second-person issues in the study of consciousness (169-178). Charlottesville: Imprint Academic.

Galán Rodríguez, Carmen. (1999). La subordinación causal y final. En Ignacio Bosque y Violeta Demonte (dir.), Gramática descriptiva de la lengua española (3597-3642). Madrid: RAE.

Gallagher, Shaun. (2012). Phenomenology. London: Palgrave-Macmillan.

Gallagher, Shaun. (2013). Coordinación y creación de sentido en la atención conjunta y la atención conjunta. Ciencias cognitivas, 3, 223-245.

García-Miguel, José María. (2006). Los complementos locativos. En Company Company, Concepción (dir.), Sintaxis histórica de la lengua española, vol.2 (pp. 1253-1336). México, D.F.: Universidad Autónoma Nacional de México.

Heine, Bernd. (1995). Conceptual grammaticalization and prediction. En John Taylor \& Robert McLaury (eds.) Language and the cognitive construal of the world (pp. 119-135). Berlin: Mouton de Gruyter.

Heine, Bernd. (1997). Cognitive foundations of grammar. Oxford: OUP.

Heine, Bernd \& Kuteva, Tania. (2002). World lexicon of grammaticalization. Cambridge: CUP.

Heine, Bernd; Claudi, Ulrike \& Hünemeyer, Friederike. (1991). Grammaticalization: A conceptual framework. Chicago: The University of Chicago Press.

Huelva Unternbäumen, Enrique (2013a). Intersubjetividad y gramática. Aspectos de una gramática fenomenológica. Frankfurt am Main, New York, Oxford: Peter Lang.

Huelva Unternbäumen, Enrique (2013b). Construcciones causativas con la preposición ante en la lengua española. Zeitschrift für romanische Philologie, 129(3), 1-27.

Husserl, Edmund. (2002). Konstitution der Intersubjektivität. En Klaus Held (ed.), Phänomenologie der Lebenswelt. Ausgewählte Texte II (pp. 166-219). Stuttgart: Reclam. [Traducido al español en Edmund Husserl. (2005). Meditaciones Cartesianas. México: Fondo de Cultura Económica. Traducción de José Gaos y Miguel García-Baro].

Hutto, Daniel. (2008). First communication: Mimetic sharing without theory of mind. En Jordan Zlatev, Timothy P. Racine, Chris Sinha \& Esa Itkonen (eds.), The shared mind. perspectives on intersubjectivity (pp. 245-276). Amsterdam \& Philadelphia: John Benjamins. 
Itkonen, Esa. (2008). The central role of normativity in language and linguistics. En Jordan Zlatev, Timothy P. Racine, Chris Sinha \& Esa Itkonen (eds.), The shared mind. perspectives on intersubjectivity (pp. 279-305). Amsterdam \& Philadelphia: John Benjamins.

Meyer-Lübke, Wilhelm. (1890-1906). Grammaire des langues romanes, vol. III (Syntaxe). Disponible en http://archive.org/details/grammairedesla03meye.

Pika, Simone. (2008). What is the nature of the gestural communication of great apes? En Jordan Zlatev, Timothy P. Racine, Chris Sinha \& Esa Itkonen (eds.), The shared mind. perspectives on intersubjectivity (pp. 165-186). Amsterdam \& Philadelphia: John Benjamins.

Penny, Ralph. (1993). Gramática histórica del español. Madrid: Ariel.

Sanders, Ted; Sanders, José \& Sweetser, Eve. (2009). Causality, cognition and communication: A mental space analysis of subjectivity in causal connectives. En Ted Sanders \& Eve Sweetser (ed.), Causal categories in discourse and cognition (pp. 20-59). Berlin, New York: Mouton de Gruyter.

Shibatani, Masayoshi. (2002). Some basic issues in the grammar of causation. En Shibatani, Masayoshi (ed.), The grammar of causation and interpersonal manipulation (pp. 1-22). Amsterdam/ Filadelfia: John Benjamins.

Tomasello, Michael. (1999). The cultural origins of human cognition. Cambridge: Harvard University Press.

Tomasello, Michael \& Call, Josep. (2006). Do chimpanzees know what others see - or only what they are looking at? En Susan Hurley \& Matthew Nudds (eds.), Rational animals? (pp. 371-384). Oxford: Oxford University Press.

Tomasello, Michael; Carpenter, Malinda; Call, Josep; Behne, Tanya \& Moll, Henrike. (2005). Understanding and sharing intentions: The origins of cultural cognition. Behavioral and Brain Sciences, 28, 675-691.

Trevarthen, Colwyn. (1979). Communication and cooperation in early infancy: A description of primary intersubjectivity. En Margaret Bullowa (ed.), Before speech (pp. 321-348). Cambridge: Cambridge University Press.

Trevarthen, Colwyn. (1980). The foundations of intersubjectivity. En David Olson (ed.), The social foundations of language and thought (pp. 216-242). New York: Norton.

Trevarthen, Colwyn. (1998). The concept and foundations of infant intersubjectivity. En Stein Bråten (ed.), Intersubjective communication and emotion in early ontogeny (pp. 15-46). Cambridge: Cambridge University Press.

Zlatev, Jordan. (2007). Intersubjectivity, mimetic schemas and the emergence of language. Intellectica, 46-47, 123-152.

Zlatev, Jordan. (2008). The co-evolution of intersubjectivity and bodily mimesis. En Jordan Zlatev, Timothy P. Racine, Chris Sinha \& Esa Itkonen (eds.), The Shared Mind. Perspectives on Intersubjectivity (pp. 215-244). Amsterdam \& Philadelphia: John Benjamins. 